University of Nebraska - Lincoln

DigitalCommons@University of Nebraska - Lincoln

Faculty Publications from the Department of Electrical \& Computer Engineering, Department Electrical and Computer Engineering

3-27-2007

\title{
Enhanced Raman scattering by self-assembled silica spherical microparticles
}

\author{
Kaijun Yi \\ University of Nebraska-Lincoln, kjyi1@bigred.unl.edu \\ H. Wang \\ University of Nebraska-Lincoln \\ Yongfeng Lu \\ University of Nebraska-Lincoln, ylu2@unl.edu \\ Z. Y. Yang \\ University of Nebraska-Lincoln
}

Follow this and additional works at: https://digitalcommons.unl.edu/electricalengineeringfacpub

Part of the Electrical and Computer Engineering Commons

Yi, Kaijun; Wang, H.; Lu, Yongfeng; and Yang, Z. Y., "Enhanced Raman scattering by self-assembled silica spherical microparticles" (2007). Faculty Publications from the Department of Electrical and Computer Engineering. 87.

https://digitalcommons.unl.edu/electricalengineeringfacpub/87

This Article is brought to you for free and open access by the Electrical \& Computer Engineering, Department of at DigitalCommons@University of Nebraska - Lincoln. It has been accepted for inclusion in Faculty Publications from the Department of Electrical and Computer Engineering by an authorized administrator of DigitalCommons@University of Nebraska - Lincoln. 


\title{
Enhanced Raman scattering by self-assembled silica spherical microparticles
}

\author{
K. J. Yi, H. Wang, Y. F. Lu, ${ }^{\text {a) }}$ and Z. Y. Yang \\ Department of Electrical Engineering, University of Nebraska-Lincoln, Lincoln, Nebraska 68588-0511
}

(Received 23 August 2006; accepted 20 December 2006; published online 27 March 2007)

\begin{abstract}
A technique was developed to achieve enhanced Raman scattering of the silicon photon modes using closely packed micro- and submicron silica spherical particles. Investigation on the particle-size dependence of Raman enhancement revealed that the strongest enhancement occurs when the particle diameter is equal to the spot size of the incident laser beam. Calculations using the OPTIWAVE $^{\mathrm{TM}}$ software based on the finite difference time domain algorithm under the perfectly matched layer boundary conditions were carried out. The results showed that photonic nanojets are formed in the vicinity outside the particles along the propagation direction of incident light. It was found that the nanojets are confined to a length of $100 \mathrm{~nm}$ with a waist of $120 \mathrm{~nm}$. The presence of the strongly localized electromagnetic fields within the nanojets accounts for the enhanced Raman scattering. This technique has potential applications both in modern and traditional areas of surface science such as surface oxidation, adhesion, corrosion, and catalytic processes, and many other areas in biology, chemistry, materials science, and microelectronics. (C) 2007 American Institute of Physics. [DOI: 10.1063/1.2450671]
\end{abstract}

\section{INTRODUCTION}

Due to small interactive cross sections $\left(10^{-30}-10^{-25} \mathrm{~cm}^{2} /\right.$ molecule), the Raman scattering signal is notoriously weak and hardly detectable. ${ }^{1}$ Extensive studies, therefore, have been conducted in order to improve the sensitivity of Raman scattering for the last two decades. ${ }^{2-14} \mathrm{Sev}$ eral mechanisms related to physics, chemistry, and optics can be used to achieve this goal, such as electromagnetic and chemical mechanisms for surface-enhanced Raman scattering (SERS), ${ }^{2-5}$ interference mechanism for interferenceenhanced Raman scattering (IERS), ${ }^{6,7}$ angle-dependent reflection for total internal reflection Raman spectroscopy (TIRRS),${ }^{8}$ resonance effect from microstructures or subwavelength particles for resonance Raman scattering (RRS), ${ }^{9,10}$ near-field optics for high-resolution near-field Raman microscopy (NORM), ${ }^{11}$ and the localized evanescent electric field and surface plasmon polariton for tip-enhanced Raman spectroscopy (TERS). ${ }^{12-15}$ Metal particles, e.g., Ag and $\mathrm{Au}$, or substrates with roughness as sample carriers are the best candidates for the realization of SERS due to the high enhancement factor $\left(10^{4}-10^{14}\right)$; however, they are irreproducible and unpredictable.

Previous studies on light scattering by microparticles predicted its potential for Raman spectroscopy. ${ }^{16-19}$ However, no further investigation has been reported to authenticate this hypothesis. In this study, the enhancement factor of Raman signals, in the order as high as $10^{4}$, was realized using closely packed silica spherical microparticles.

The microparticle-enhanced Raman spectroscopy has at least three advantages over the conventional SERS approaches. First, the location and morphology of dielectric microparticles can be well controlled, eliminating the irre-

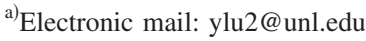

producibility in SERS. Second, the sample preparation process is much simpler, avoiding tedious efforts in substrate preparation. Third, the enhanced effects by microparticles can occur for most Raman-active substrates such as silicon.

Calculations on the effect of spherical dielectric microparticles on incident plane waves and focused Gaussian beams indicated that the spatial distribution of the electromagnetic intensity can be changed both within and outside the microparticles. The curved surfaces of the particles and discontinuity in the refractive index at the interfaces result in the changes of the electromagnetic field distributions. This phenomenon has been confirmed using the fluorescence from iodine vapor to image the near-field intensity distribution and the fluorescence from ethanol droplets impregnated with rhodamine 590 to visualize the internal distribution. ${ }^{17} \mathrm{Re}-$ cently, localized photonic nanojets generated at shadow surfaces of microscale cylinders illuminated by a plane wave was reported..$^{20}$ These nanojets have a waist smaller than the diffraction limit and can extend over several optical wavelengths without significant diffraction. Coupled resonator optical waveguides might be built from the chains of dielectric microspheres through two mechanisms in optical transport, namely, evanescent coupling and nanojet coupling. ${ }^{21}$ The electromagnetic fields in the nanojets are much stronger than those in other areas. Therefore, the Raman scattering could be enhanced since it is strongly dependent on the magnitude of the electromagnetic field. ${ }^{22}$

\section{EXPERIMENT AND SIMULATION}

Silicon substrates covered with a monolayer of silica microparticles were prepared by a self-assembly method. First, silicon substrates, cleaved into $10 \times 10 \mathrm{~mm}^{2}$ pieces along the crystal direction, were cleaned by ultrasonic agitation in methanol, acetone, and de-ionized water for $5 \mathrm{~min}$ each. The prepared substrates were then placed into a bath of freshly 


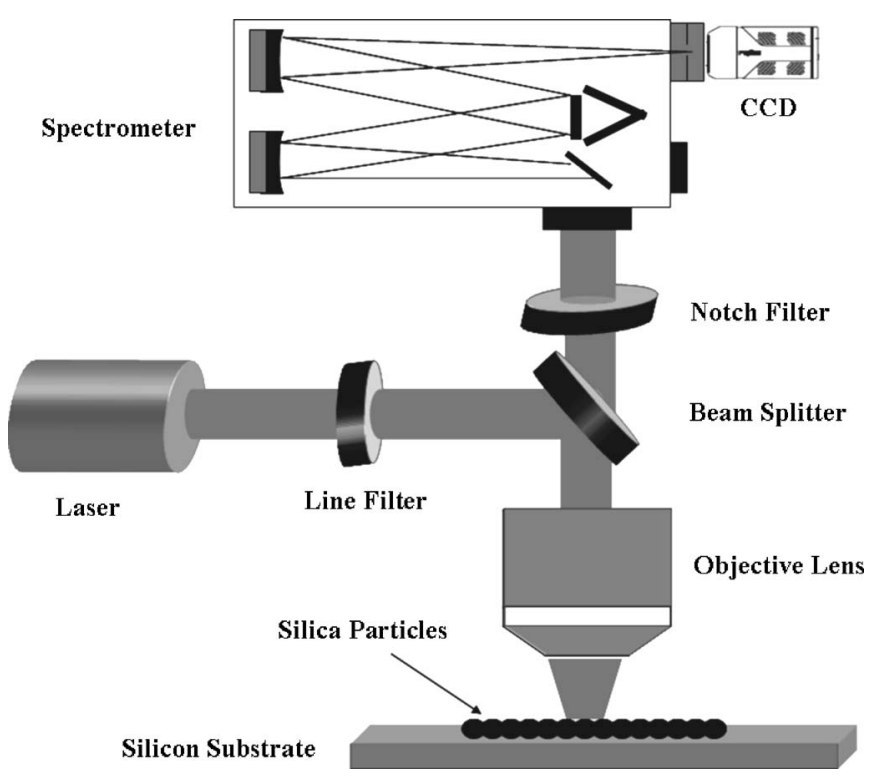

FIG. 1. Experiment setup of the micro-Raman spectrometer for microparticle-enhanced Raman scattering.

prepared Piranha solution $\left(\mathrm{H}_{2} \mathrm{SO}_{4} / \mathrm{H}_{2} \mathrm{O}_{2}=7: 3\right)$. Hydrofluoric $(\mathrm{HF})$ acid was then used to remove the oxide film. After the substrates were rinsed thoroughly in de-ionized water and ethanol, they were ready for self-assembly of silica particles. The substrates were tilted with an angle $\left(40^{\circ}-60^{\circ}\right)$ to the base. After the surfactant was air dried, a drop of monodisperse suspensions (10\% solution of silica microparticles) was dropped on the tilted samples to obtain a hexagonally closely packed assembly of silica particles over an area of several hundred $\mu \mathrm{m}^{2}$. The surface morphologies of the samples were observed under an optical microscope (Olympus, $\mathrm{BH}-2)$ equipped with a high-resolution charge-coupled device (CCD) camera.

Enhanced Raman spectroscopy was carried out using a homemade Raman spectrometer. The instrumental setup is illustrated in Fig. 1. An argon laser (Coherent, Innova 300) with a wavelength of $514.5 \mathrm{~nm}$ was used as the excitation source. A $50 \times$ microscope objective lens [Olympus, numerical aperture $(\mathrm{NA})=0.70]$ was employed to focus the incident beam into a laser spot with a full width at half maximum (FWHM) waist of around $2.5 \mu \mathrm{m}$. Raman scattering was collected by the same lens. Most of the overwhelming Rayleigh scattering was blocked by a notch filter [SuperNotch filter, Leica, optical density $(\mathrm{OD})=6]$. A triple-grating dispersive imaging spectrograph (Princeton Instrument, SP-2300i) with a $300 \mathrm{~mm}$ focal length was used to detect the Raman signal in a wide spectral range through a thermoelectrically cooled CCD camera (Princeton Instrument, PIXIS 400B). The system is capable of scanning samples in three different modes: point mode (Raman spectra at a specific position), line mode (line scanning to locate the interested area), and zone mode (Raman mapping in a specified region). The sample was placed on a motorized motion stage with a resolution of $3.175 \mu \mathrm{m}$. A $3.15 \mathrm{~cm}^{-1}$ resolution of Raman shift can be achieved through the optical grating with a density of 600 lines/mm.

Since the silica particles did not cover the entire surface,

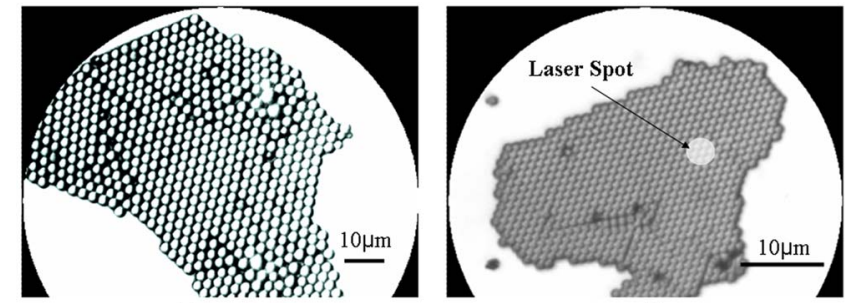

(a)

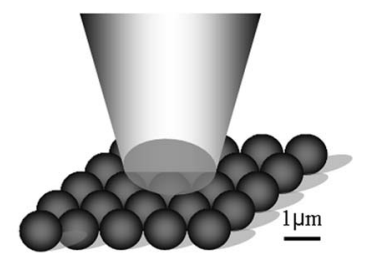

(c)

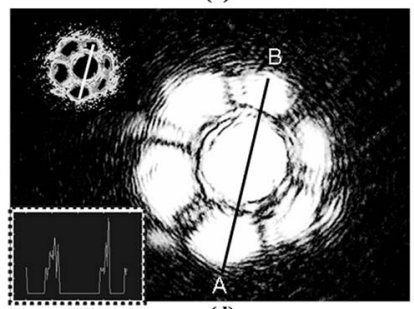

(d)
FIG. 2. (a) Surface morphology of the silicon substrate covered with the silica particles of $2.34 \mu \mathrm{m}$ diameter by optical microscope; (b) surface morphology of the silicon substrate covered with the silica particles of $1.01 \mu \mathrm{m}$ diameter by optical microscope; (c) schematic diagram of the closely packed silica particles of $1.01 \mu \mathrm{m}$ diameter illuminated by a focused laser beam; (d) image in scattering mode for the silica particles of $1.01 \mu \mathrm{m}$. The part of the enclosed area in white color in (b) indicates the incident laser beam. The bottom-left inset is the profile of (d) along the line $A B$, while the top-left inset is the contour of (d).

the samples were first measured with the line mode to locate the regions of interest. Then, the Raman spectra were recorded with the point mode. Next, a CCD camera was placed in front of the notch filter to capture the scattering images of the particles. The brightness of the camera was adjusted in order to obtain pictures with high contrast.

We used the OPTIWAVE ${ }^{\mathrm{TM}}$ software to calculate the electric fields both inside and outside the particles. The simulation was performed using the finite difference time domain (FDTD) algorithm under the perfectly matched layer (PML) boundary conditions. The physical model consisted of four components: the focused Gaussian laser beam, the surrounding medium (air), the spherical silica particles, and the silicon substrates. The propagation direction of the illuminated laser was normal to the surface of the silicon substrates. The calculation step was $10 \mathrm{~nm}$.

\section{RESULTS AND DISCUSSION}

Spherical silica particles with nine different diameters $(0.33,0.52,0.81,1.01,1.52,2.34,3.22,3.93$, and $5.08 \mu \mathrm{m})$ were employed to study the size dependence of Raman scattering. The enhancement in Raman scattering was observed for all samples. The enhancement effects were confirmed to be size dependent. The prepared substrates covered with monolayers of silica particles are shown in Figs. 2(a) $(2.34 \mu \mathrm{m})$ and 2(b) $(1.01 \mu \mathrm{m})$.

As for the particles of $1.01 \mu \mathrm{m}$ diameter, the laser spot can cover two and a half particles, as shown in the schematic diagram in Fig. 2(c). In this figure, only one particle can be entirely covered by the focused laser beam, while the six particles adjacent to it are partially covered. A large circular spot in the center and six fan-shaped spots surrounding the center spot can be clearly observed. The image is not per- 


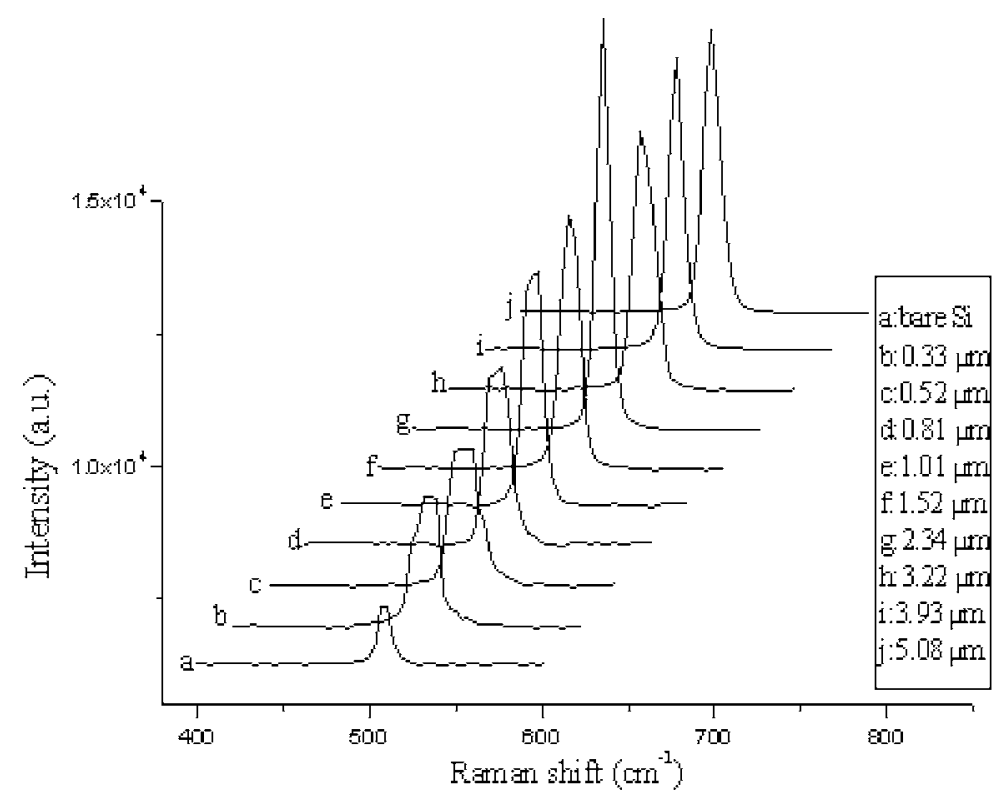

(a)

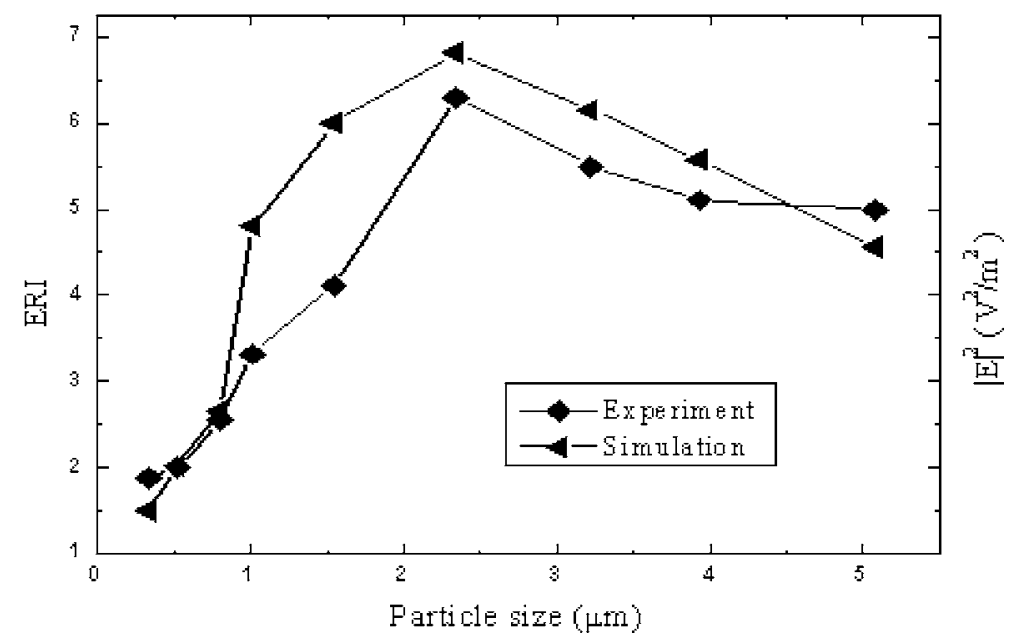

(b)
FIG. 3. (a) Raman spectra of bare silicon and silicon substrates covered with silica particles with different sizes from 0.33 to $5.08 \mu \mathrm{m}$; (b) size dependence of ERI obtained in experiments (diamonds) and $|E|^{2}$ obtained in calculations (triangles). fectly symmetrical due to the limited step resolution of the stage. The substrates covered with the particles of $1.01 \mu \mathrm{m}$ diameter exhibited the strongest Raman scattering, with the optical image shown in Fig. 2(d). The image resulted from the reflected as well as the scattered light, including Rayleigh and Raman scattering. The upper-left inset in Fig. 2(d) is the contour of the image, while the bottom-left inset gives the profile. Clearly, the image shape in the scattering mode is determined by the geometries of both the particles and the laser beam (size and beam shape). Since the particle size and beam geometry were known in the experiment, the beam size could be estimated through the observed image. In Fig. 2(d), two to three particles are contained along the line $A B$, indicating that the beam size is between 2.02 and $3.03 \mu \mathrm{m}$, presumably around $2.5 \mu \mathrm{m}$, which verified our previous estimation. Furthermore, the beam size can be figured out through the size dependence of Raman scattering, as discussed afterward.

The electromagnetic field caused by the focused laser beam is determined both by the beam parameters (wavelength, power, mode, and beam size) and the particle prop- erties (size and complex index of refraction). The Raman scattering intensity, therefore, is a function of the combination of both the incident beam and the particles. The Raman spectra of the bare silicon and the silicon substrates covered with the silica microparticles of different sizes (from 0.33 to $5.08 \mu \mathrm{m})$ are shown in Fig. 3(a). The spectrum of bare silicon was also measured for reference. The sharp Raman peak around $520 \mathrm{~cm}^{-1}$ can be observed. A certain offset was added to each curve for clarity. The Raman signals for all substrates were enhanced to some extent. In the calculation of the Raman signal intensity, the three highest points near the peak were averaged and the background signal was subtracted. The obtained data were applied to calculate the enhancement of Raman intensity (ERI), defined as the ratio of the net enhanced Raman intensity of the silicon substrates (with background subtraction) covered with microparticles to the net intrinsic Raman intensity from the reference bare silicon (with background subtraction). However, in order to calculate the enhancement factor, the scattering volume must be considered. The optical penetration depth in silicon in the far field is around $500 \mathrm{~nm}$. Then the far-field volume was calcu- 


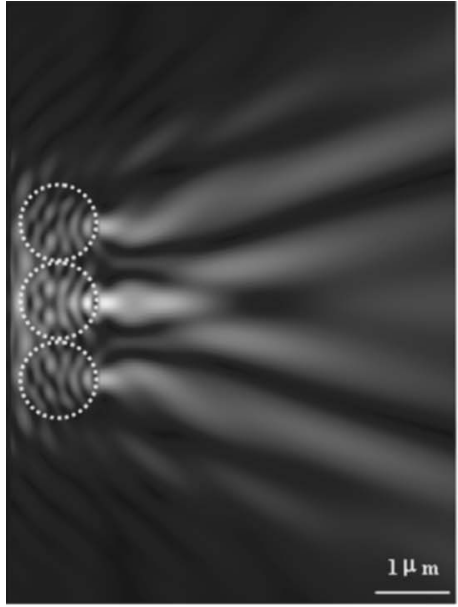

(a)

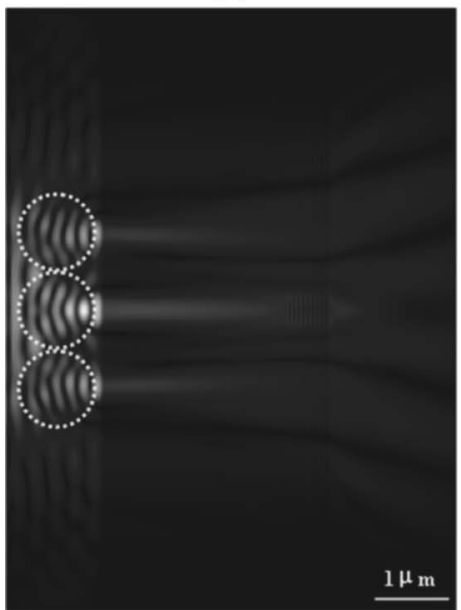

(c)

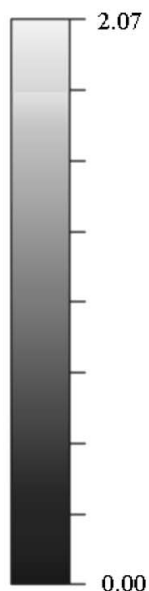

0.00

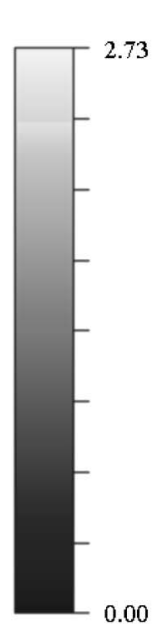

0.00

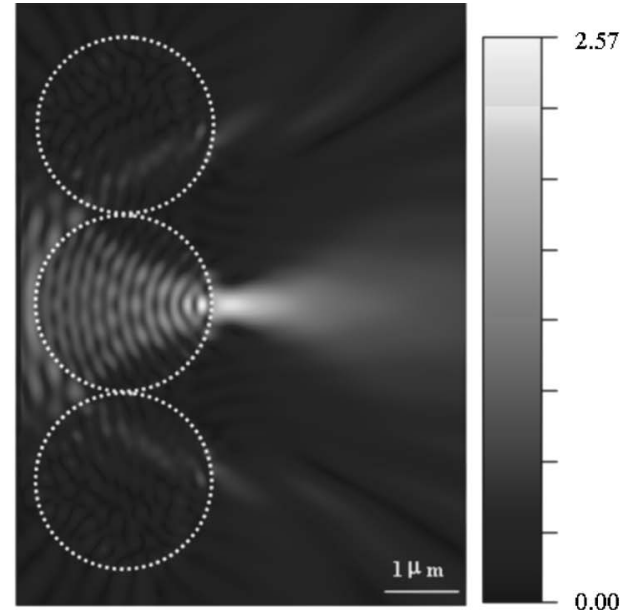

(b)

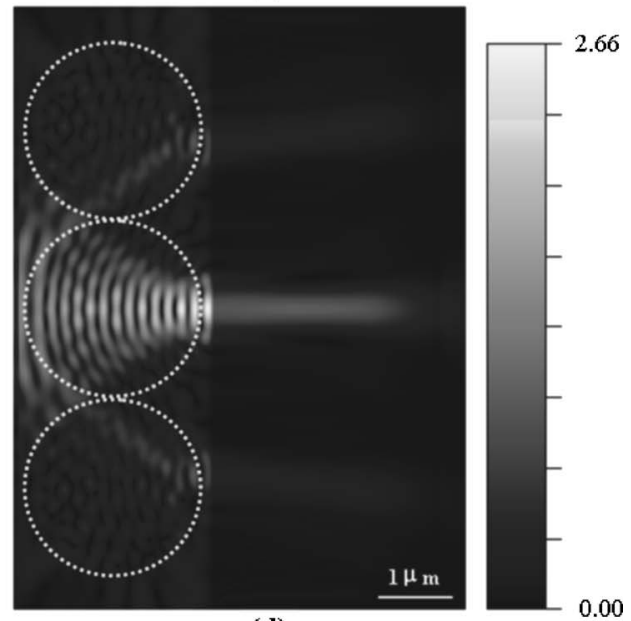

(d)
FIG. 4. Calculated distributions of the electric fields for the different sizes of particles with and without the substrates from the $X Z$ cross-sectional view using the software OPTIWAVE ${ }^{\mathrm{TM}}$ : (a) the particles of $1.01 \mu \mathrm{m}$ without substrate; (b) the particles of $2.34 \mu \mathrm{m}$ without substrate; (c) the particles of $1.01 \mu \mathrm{m}$ with substrate; and (d) the particles of $2.34 \mu \mathrm{m}$ with substrate. The area in the white circle indicates the location of the particle. lated as $2.45 \mu \mathrm{m}^{3}$. The penetration depth in the near field is $100 \mathrm{~nm}$ (calculated value). The diameter of the beam in near field is around $120 \mathrm{~nm}$ (calculated value) for the $2.34 \mu \mathrm{m}$ particles. Thus, the near-field volume is around 1.13 $\times 10^{-3} \mu \mathrm{m}^{3}$. Thus, the enhancement factor for the $2.34 \mu \mathrm{m}$ particle was calculated to be as high as $1.4 \times 10^{4}$.

Figure 3(b) shows the size dependence of the ERI obtained experimentally (diamonds) and the size dependence of $|E|^{2}$ (squared electrical field) obtained by simulation (triangles). For the particles with sizes smaller than $2.34 \mu \mathrm{m}$, the ERI increases continuously with the increase in particle size, but starts to decrease as the particle size becomes larger than $2.34 \mu \mathrm{m}$. The maximum ERI at $2.34 \mu \mathrm{m}$ might indicate that this diameter is close to the waist of the incident laser beam $(2.5 \mu \mathrm{m})$. This observation is consistent with the previous analysis on the beam diameter based on the particle image in scattering mode. Thus, the particle size can be optimized to achieve the strongest enhancement under different optical conditions.

Because the particle sizes are comparable with the incident wavelength, silica particles can be used to generate near-field effects. ${ }^{23}$ The intensity of the Raman scattering is proportional to the square of the magnitude of the electromagnetic fields incident on the sample. Using the OPTIWAVE $^{\mathrm{TM}}$ software, we calculated the electrical field intensity with single- and multiple-particle models. The incident laser beam had a Gaussian shape with a FWHM waist of $2.5 \mu \mathrm{m}$. The amplitude of the electric field was assumed to be $1 \mathrm{~V} / \mathrm{m}$. The refractive indices of air, silica, and silicon at the wavelength of $514.5 \mathrm{~nm}$ are $1.0,1.5$, and 4.24 , respectively. The extinction coefficient of silicon is 0.06 . All optical constants are quoted from Ref. 24. The light propagates along the $Z$ direction, normal to the substrate surface. The incident laser is polarized in the $Y$ direction (not indicated in the figures). The calculated electric fields for the particle sizes of 1.01 and $2.34 \mu \mathrm{m}$ without the silicon substrates are shown in Figs. 4(a) and 4(b), respectively. The figures are visualized from the $X Z$ cross-sectional view. It can be clearly observed that the intensities inside the particles are not uniformly distributed. The electric fields are focused in the forward direction along the incident light near the shadow surface. Nanojets are generated and the waist of the nanojets in Figs. 4(a) and 4 (b) are almost the same $(120 \mathrm{~nm})$, which is the same as the results reported by Ref. 20. This phenomenon indicates that the nanojet waist only depends on the incident wavelength. On the other hand, there is a great difference on the length of the nanojets for different particles. The strongest light intensity occurs inside the particles in Fig. 4(a), while it appears outside the particles in Fig. 4(b). This is due to the greater internal reflection resulting from the inner side of the shadow surface of the particles of $2.34 \mu \mathrm{m}$ diameter compared to that of the particles of $1.01 \mu \mathrm{m}$ diameter. 
The calculated electric fields for the particles of 1.01 and $2.34 \mu \mathrm{m}$ diameter positioned on silicon substrates are shown in Figs. 4(c) and 4(d). The distributions of the electric fields are greatly changed due to the high refractive index of silicon. Interestingly, even though the beam waists are not changed, the length of nanojets becomes much smaller, shrinking into about $100 \mathrm{~nm}$. This finding indicates that the electric field is well confined to the surface of the substrates in a nanometer-scaled region. The size dependence of the simulated $|E|^{2}$, to which near-field optical intensity is directly related, is shown in Fig. 3(b) (triangles). The simulated curve also shows the peak at $2.34 \mu \mathrm{m}$, consistent with the experimental results in Raman spectroscopy.

\section{CONCLUSIONS}

We prepared silicon substrates covered with spherical silica microparticles to study the particle-enhanced Raman spectroscopy. It is observed that the Raman peaks of silicon could be significantly enhanced with the presence of dielectric microparticles. Simulation results showed that the enhancement effects come from the localized electric fields within the nanojets in the vicinity outside the particles along the propagation direction of the incident laser. The optical nanojets were formed by the silica particles, with a length as small as $100 \mathrm{~nm}$ and a waist of $120 \mathrm{~nm}$ for the particles of $2.34 \mu \mathrm{m}$ diameter. The dimensions of the nanojects are smaller than the optical diffraction limit. The presence of the nanojets is believed to be the primary reason to cause the particle-enhanced Raman scattering.

\section{ACKNOWLEDGMENTS}

This work was financially supported by the National Science Foundation (Nos. DMI 0555884 and ECS 0629280),
Nebraska Research Initiative, and Research Foundation for Opto-Science and Technology (Japan).

${ }^{1}$ M. Futamata, Meas. Sci. Technol. 2, 1207 (1991).

${ }^{2}$ M. Moskovits, J. Raman Spectrosc. 36, 485 (2005).

${ }^{3}$ W. Song et al., J. Raman Spectrosc. 37, 755 (2005).

${ }^{4}$ E. J. Ayars and H. D. Hallen, Appl. Phys. Lett. 76, 3911 (2000).

${ }^{5}$ P. Etchegoin, L. F. Cohenb, H. Hartigan, R. J. C. Brown, M. J. T. Milton, and J. C. Gallop, J. Chem. Phys. 119, 5281 (2003).

${ }^{6}$ G. A. N. Connell, R. J. Nemanich, and C. C. Tsai, Appl. Phys. Lett. 36, 31 (1979).

${ }^{7}$ C. M. Dai, D. S. Chuu, and W. F. Hsieh, Appl. Phys. Lett. 59, 3273 (1991).

${ }^{8}$ R. Iwamoto, M. Miya, K. Ohta, and S. Mima, J. Chem. Phys. 74, 4780 (1981).

${ }^{9}$ D. V. Murphy and S. R. J. Brueck, Opt. Lett. 8, 494 (1983).

${ }^{10}$ L. A. Kuzik, V. A. Yakovlev, and G. Mattei, Appl. Phys. Lett. 75, 1830 (1999).

${ }^{11}$ C. L. Jahncke, H. D. Hallen, and M. A. Paesler, J. Raman Spectrosc. 27, 579 (1998).

${ }^{12}$ W. X. Sun and Z. X. Shen, Mater. Phys. Mech. 4, 17 (2001).

${ }^{13}$ W. X. Sun and Z. X. Shen, J. Raman Spectrosc. 34, 668 (2003).

${ }^{14}$ N. Anderson, A. Bouhelier, and L. Novotny, J. Opt. A, Pure Appl. Opt. 8, 227 (2006).

${ }^{15}$ Y. Iwanabe, M. Fujimaki, K. Awazu, T. Horiuchi, and J. Tominaga, Nanotechnology 17, 1717 (2006).

${ }^{16}$ J. P. Barton, D. R. Alexander, and S. A. Schaub, J. Appl. Phys. 64, 1632 (1988).

${ }^{17}$ D. S. Benincasa, P. W. Barber, J. Z. Zhang, W. F. Hsieh, and R. K. Chang, Appl. Opt. 26, 1348 (1987).

${ }^{18}$ C. L. Adler, J. A. Lock, B. R. Stone, and C. J. Garcia, J. Opt. Soc. Am. A 14, 1305 (1997).

${ }^{19}$ J. A. Lock, C. L. Adler, and E. A. Hovenac, J. Opt. Soc. Am. A 17, 1846 (2000).

${ }^{20}$ Z. Chen, A. Taflove, and V. Backman, Opt. Express 12, 1214 (2004).

${ }^{21}$ Z. Chen, A. Taflove, and V. Backman, Opt. Lett. 31, 389 (2006).

${ }^{22}$ C. Li, G. W. Kattawar, P.-W. Zhai, and P. Yang, Opt. Express 13, 4554 (2005).

${ }^{23}$ J. R. Arias-González and M. Nieto-Vesperinas, Opt. Lett. 25, 782 (2000).

${ }^{24}$ Handbook of Optical Constants of Solids, edited by E. D. Palik (Academic, Orlando, FL, 1985). 